\title{
Dictynna
}

Dictynna

Revue de poétique latine

$18 \mid 2021$

Varia

\section{Non ego te: breve storia di uno stilema patetico}

\section{Paolo Dainotti}

\section{(2) OpenEdition}

\section{Journals}

\section{Edizione digitale}

URL: https://journals.openedition.org/dictynna/2523

DOI: $10.4000 /$ dictynna.2523

ISSN: $1765-3142$

\section{Editore}

Université de Lille

\section{Notizia bibliografica digitale}

Paolo Dainotti, « Non ego te: breve storia di uno stilema patetico », Dictynna [En ligne], 18| 2021, mis en ligne le 01 décembre 2021, consulté le 18 décembre 2021. URL : http://journals.openedition.org/ dictynna/2523; DOI : https://doi.org/10.4000/dictynna.2523

Questo documento è stato generato automaticamente il 18 décembre 2021.

\section{(c) (i) (9)}

Les contenus des la revue Dictynna sont mis à disposition selon les termes de la Licence Creative Commons Attribution - Pas d'Utilisation Commerciale - Pas de Modification 4.0 International. 


\title{
Non ego te: breve storia di uno stilema patetico ${ }^{1}$
}

\author{
Paolo Dainotti
}

1 Nella storia della poesia può capitare che una sequenza di parole particolarmente efficace catturi l'attenzione di un lettore-poeta, che la ripropone nella sua poesia, di fatto riconoscendole lo status della letterarietà. La sequenza diviene così uno stilema, un contrassegno stilistico immediatamente riconoscibile da un lettore colto, il quale sarà in grado anche di percepire l'effetto della citazione. Se sintagmi particolarmente arditi s'impongono, come è ovvio, facilmente all'attenzione, non è tuttavia lo stesso per sequenze di parole più neutre, le quali rischiano, almeno per il lettore meno scaltrito, di passare inosservate. È questo, ad esempio, il caso della sequenza non ego te, un ritmema - in quanto nell'esametro è collocata sempre ad inizio di verso - che costituisce anche un elegante stilema patetico, come vorrei dimostrare in questo mio articolo, nel quale analizzerò le varie occorrenze seguendo un andamento cronologico (da Plauto fino all'ultima attestazione in Ausonio).

2 Quando si vuole tentare di interpretare una figura stilistica, operazione sempre in qualche modo rischiosa, è bene in via preliminare considerare alcuni dati statistici che possano rilevarne la rarità e far luce su eventuali ambiti di impiego. È, ad esempio, sintomatico che delle 40 occorrenze totali della sequenza non ego te solo due appartengano alla prosa (e in contesti alti), un dato, questo, che conferma che il sintagma è chiaramente poetico. ${ }^{2}$ Delle restanti 38 occorrenze ben 13 appartengono alla commedia (12 in Plauto, 1 in Afranio), ${ }^{3}$ ma l'assenza del sintagma in Terenzio sembrerebbe indicare che più che un tratto del parlato esso sia piuttosto un elemento peculiare della lingua di Plauto. Per quanto riguarda il sintagma, va, inoltre, aggiunto che esso non deve essere identificato con la semplice giustapposizione pronominale ego te, un ordo verborum espressivo che è del resto particolarmente ricorrente nella lingua latina, ${ }^{4}$ né con l'incipit esametrico non ego. ${ }^{5}$

3 Veniamo adesso all'analisi dei contesti. In alcuni casi la sequenza non ego te è associata alle forme verbali novi e vidi in interrogative retoriche che presuppongono una risposta affermativa e suonano come accuse stringenti ('Non ti ho forse visto?', 'Forse che non ti 
conosco?'). Si pensi ad esempio a Plaut. Amph.518 Carnufex, non ego te novi? Abin e conspectu meo? ('Boia, credi che io non sappia chi sei? Non ti allontani dalla mia presenza?'); Men.511 s. non ego te indutum foras / exeire vidi pallam? ('Come! non t'ho visto uscire da casa tua drappeggiato in questo mantello?'); Capt.563 s. At etiam, furcifer, / male loqui mi audes? Non ego te novi? ('Perciò, avanzo di forca, osi ancora sparlare di me? io non ti conoscerei?'); o ancora a Men.632 ss. Non ego te modo hic ante aedis cum corona florea / vidi astare, quom negabas mihi esse sanum sinciput / et negabas me novisse, peregrinum aibas esse te? ('Non t'ho visto poco fa, sulla porta di quella casa, in piedi, con una corona di fiori in testa, quando dicevi ch'io ero ammalato nella cervice e negavi di conoscermi e asserivi di essere un forestiero?'), dove l'accusa è tutta un ragionamento serrato. ${ }^{6}$

Ma l'esempio più significativo per la nostra indagine è Plaut. Amph.531 Non ego te hic lubens relinquo neque abeo abs te ('Non ti lascio, non me ne vado per mio piacere'), nelle languide parole di giustificazione di Giove, che, dopo un'interminabile notte d'amore, prova a congedarsi dall'insaziabile Alcmena. ${ }^{7}$ In questo caso la sequenza converge con una particolare apostrofe - gli antichi la chiamavano aversio - consistente nell'anticipare, negandola, un'accusa che può essere mossa dall'interlocutore. ${ }^{8}$

5 È proprio quest'ultima occorrenza, comicamente solenne e stilisticamente ricercata (si noti la litote giustificatoria non ... lubens), a transitare nella lingua dell'epos mediante il significativo riuso di Cat.64.212 ss.

namque ferunt olim, classi cum moenia diuae

linquentem gnatum uentis concrederet Aegeus,

talia complexum iuueni mandata dedisse:

'gnate mihi longa iucundior unice uita,

gnate, ego quem in dubios cogor dimittere casus, reddite in extrema nuper mihi fine senectae, quandoquidem fortuna mea ac tua feruida uirtus eripit inuito mihi te, cui languida nondum lumina sunt gnati cara saturata figura, non ego te gaudens laetanti pectore mittam, nec te ferre sinam fortunae signa secundae, sed primum multas expromam mente querellas, canitiem terra atque infuso puluere foedans,

('Dicono infatti che, affidando ai venti il figlio che lasciava con nave la città della dea, Egeo dopo l'abbraccio gli diede quest'ordine: "Mio solo figlio, a me più caro della lunghissima vita, figlio, che ora devo affidare a destino malcerto dopo che t'ho ritrovato sul finire della vecchiaia, poiché la mia sorte e il tuo valore irruento a me contrario ti strappano, che gli occhi languenti ancora non ho saziato della tua cara figura, io non ti lascio partire con animo lieto e non permetto che porti i segni del successo ma prima voglio dal cuore liberare molti lamenti, lordando la bianca chioma di terra e di polvere sparsa'. Trad. N. Gardini)

6 L'allusione, finora inosservata, ${ }^{9}$ è evidente, oltre che per il sintagma non ego te in posizione incipitaria, anche per l'uso del participio presente gaudens, una variatio isosillabica (ma non isoprosodica) e semanticamente affine al plautino lubens. Il poeta veronese seleziona il modello plautino per via della similarità di situazione, il commiato 
(mittam richiama, variandoli, i verbi relinquo e abeo), aggiungendovi però, in un'elegante oppositio in imitando, un tono e un tocco marcatamente patetici: se Anfitrione, appagato, si congeda dalla sua amante, Egeo, con ben altri sentimenti, è costretto a lasciar partire l'audace figlio verso un'impresa irta di pericoli.

Il passo, oltre a presentare in nuce alcuni motivi che saranno ricorrenti nella poesia di Virgilio, come il pathos ab aetate (Teseo è iuuenis, mentre Egeo è ormai sul limitare della morte $)^{10} \mathrm{e}$ il rapporto padre-figlio, ${ }^{11}$ è caratterizzato da alcune forme dell'espressione particolarmente efficaci ad esprimere il pathos. È questa la funzione dell'anafora dell'affettivo gnate, ${ }^{12}$ delle giustapposizioni ad effetto (ego quem ... mea ac tua ... mihi te ... ego te), che segnano lo stretto legame tra Egeo e Teseo, dell'emotivo eripio, che dice la violenza del distacco, dei pleonasmi (in extrema fine ... gaudens laetanti), ${ }^{13}$ del sintagma poetico languida lumina,$^{14}$ e della litote (non ... gaudens), la quale evidenzia che la gioia dell'amore paterno è evocata solo per contrasto, è una gioia negata. ${ }^{15} \mathrm{Il}$ vecchio Egeo si vede costretto a lasciare andare il figlio, ma, preso da un triste presentimento, non permette la navigazione con le vele bianche, in quanto sarebbero segno di felicità; già piange e, come in segno di lutto, si cosparge i capelli di terra e polvere. ${ }^{16}$

Lo stilema è ancora impiegato da Catullo, ma leggermente variato (con il più espressivo numquam in luogo della semplice negazione non), e ad inizio di pentametro, in un passo fortemente patetico, 65.10 ss.:

numquam ego te, vita frater amabilior,

aspiciam posthac? at certe semper amabo,

semper maesta tua carmina morte canam,

('io te, fratello mio, non rivedrò,

caro più della vita? Ma sempre ti amerò,

sempre ti canterò lugubre canto'. Trad. N. Gardini)

Il poeta 'scrive' ad Ortalo, che lo ha invitato a comporre versi, e, mentre sta rivelando di essere riuscito, solo con molta difficoltà, a tradurre, piuttosto che a comporre, poiché la sua vena poetica era stata annullata dalla morte del fratello, con una toccante apostrofe si rivolge direttamente al caro defunto. ${ }^{17}$ La sintassi spezzata, per via della lunga parentetica che occupa la parte centrale della composizione, un efficace «ỏ $\mu \varphi \alpha \lambda$ ó patetico del discorso" ${ }^{18}$ tradisce tutta l'emozione di Catullo, che, ancora incredulo, chiede al fratello, che gli è più caro della sua stessa vita - si noti la similarità linguistica e di situazione con il passo citato dal carme $64-$, se sia mai possibile che non lo rivedrà più. Questo motivo patetico del 'non ti rivedrò più', con la sequenza non ego te associata ad un verbum videndi, come vorrei mostrare, godrà di una certa fortuna anche nella poesia successiva.

10 È superfluo ricordare la centralità di Catullo nella formazione della lingua poetica, un poeta che, in quanto a fatti di stile, fa spesso da trait d'union tra commedia e poesia augustea, ${ }^{19} \mathrm{e}$ al quale Virgilio attinge soprattutto in passi particolarmente patetici. ${ }^{20}$

11 Il nesso, quindi, grazie all'autorità del Catullo dei carmina docta, è ormai possesso anche della poesia più alta. Virgilio lo impiega, ad esempio, in tre casi, uno per ogni opera, con modalità espressive, toni e registri che rispecchiano le differenze stilistiche delle opere virgiliane. La prima attestazione è nella terza bucolica (vv.17 s.), una composizione che, come è noto, è intrisa di elementi plautini sia nella lingua sia nella situazione (la disputa tra due schiavi): ${ }^{21}$ 
non ego te uidi Damonis, pessime, caprum

excipere insidiis multum latrante Lycisca?

('Non ti ho veduto io, furfante, fare la posta

al capro di Damone, mentre latrava Licisca?') ${ }^{22}$

12 Virgilio, nel ricalcare il linguaggio humilis della commedia, impiega la sequenza non ego te in una modalità simile ai più comuni passi plautini, ovvero all'interno di una domanda retorica con valore d'accusa: Menalca, nell'accusare Dameta di aver sottratto il capro di Damone, gli ricorda di averlo visto di persona (uidi). Il modello plautino è stranamente sfuggito ai commentatori di Virgilio, i quali non mancano però di indicare altri termini che rimandano chiaramente al sermo di Plauto, come, ad esempio, il vocativo pessime, impiegato da Virgilio solo in questo passo. ${ }^{23}$ Il linguaggio è, invece, sensibilmente più alto in georg.2.101 $\mathrm{s}$.

non ego te, dis et mensis accepta secundis,

transierim, Rhodia, et tumidis, bumaste, racemis.

('E come potrei dimenticare il vino di Rodi,

così gradito a brindisi e a dei,

o l'uva pergola dai grappoli rigonfi?') ${ }^{24}$

Il poeta, con un elegante gesto leggermente parodico, impiega una litote 'panegiristica' ('non potrei tralasciare te') ${ }^{25}$ in un contesto inusuale, all'interno di un catalogo di piante. Il tono del passo è alto, come appare evidente da tutta una serie di figure stilistiche che convergono con la litote, quali la doppia apostrofe (Rhodia, bumaste), l'efficace enjambement della forma verbale transierim messa in rilievo dalla tritemimera, l'apposizione (dis et mensis accepta secundis), lo zeugma (dis et mensis), la ricercata collocazione del vocativo tra due termini ad esso correlati (tumidis, bumaste, racemis), ${ }^{26}$ il raffinato gioco di parole (tumidis racemis è elegante glossa di bumaste) ${ }^{27} \mathrm{e}$ l'ampia posposizione del vocativo (te ... Rhodia). Virgilio, insomma, con una movenza propria dell'epos si rivolge alle due piante quasi fossero condottieri o guerrieri di un catalogo. ${ }^{28}$

14 La letterarietà del sintagma è evidente se accostiamo al passo appeno commentato Aen. 10.185 ss.

Non ego te, Ligurum ductor fortissime bello, transierim, Cunere, et paucis comitate Cupauo, cuius olorinae surgunt de uertice pinnae, crimen, Amor, uestrum formaeque insigne paternae.

('Non te potrei tralasciare, eroico comandante guerriero dei Liguri, o Cunaro, e te, o Cupavone, da pochi accompagnato, dal cui cimiero spuntano penne di cigno, vostro indizio, Amore, e insegna della bellezza paterna.')

dove, in un chiaro esempio di auto-citazione, ${ }^{29}$ Virgilio impiega in un contesto simile ancora un catalogo ma ovviamente non ironico, dato che il panegirico è rivolto a due guerrieri - la stessa sequenza, sempre con elegante litote e doppia apostrofe (ai personaggi di Cunerus e Cupauo) $)^{30}$ e la medesima forma verbale transierim in enjambement. Nell'Eneide il tono del sintagma è ovviamente più solenne, inserito com'è in un genere e in un contesto più alto, in un periodo che si dispiega per quattro versi ed è ulteriormente impreziosito da altri accorgimenti stilistici, come ad esempio l'efficace allitterazione Cunere... comitate Cupauo, la quale seleziona i nomi dei due guerrieri, 
legandoli non a caso al vocativo comitate, che sottolinea l'idea della vicinanza e dell'unione.

Accanto a questi esempi meritano di essere citati altri passi virgiliani nei quali la sequenza, sebbene leggermente variata, presenti i medesimi effetti stilistici. È questo il caso di b.1.74 ss.

ite meae, felix quondam pecus, ite capellae.

non ego uos posthac uiridi proiectus in antro

dumosa pendere procul de rupe uidebo,

carmina nulla canam; non me pascente, capellae,

florentem cytisum et salices carpetis amaras.

('Andate, mio gregge un tempo felice, andate, caprette:

non io d'ora innanzi, disteso in una verde grotta,

vi vedrò sospese ai cespugli di una rupe lontana;

non canterò più canzoni; non me pastore, caprette,

brucherete il trifoglio in fiore e i salici amari.')

dove la formula, variata in non ego uos (unica occorrenza in Virgilio) ${ }^{31}$ e associata ad un verbum videndi (uidebo), come in Cat.65.11 (aspiciam), ${ }^{32}$ esprime il motivo patetico del 'non ti rivedrò più'. La citazione riportata è più ampia rispetto al singolo periodo al quale appartiene il sintagma, in quanto il contesto, come spesso accade, permette di misurare meglio la figura in tutti i suoi effetti e in tutta la sua carica patetica. I versi riportati costituiscono, come è ben noto, le ultime parole di Melibeo, che, ormai esule, si congeda dal suo mondo. Il primo verso ben esemplifica quel concetto di 'convergenza di fattori stilistici' che deve essere alla base di ogni corretta interpretazione stilistica: non è mai una singola figura ad elicitare un effetto, ma è sempre un concentus, una convergenza di più fattori - semantica, metro, sintassi ed altre figure - a caratterizzare in senso espressivo la dizione. ${ }^{33} \mathrm{Al}$ primo verso il pathos espresso mediante la ripetizione, in posizioni marcate (a inizio di verso e dopo dieresi bucolica) dell'imperativo ite ${ }^{34}$ - più che di anafora in questo caso si dovrebbe parlare di separatio, una particolare forma di geminatio con una parola (o più parole) interposta $-{ }^{35}$ è ulteriormente evidenziato dall'ampio iperbato meae ... capellae (il possessivo e il diminutivo hanno entrambi valore affettivo), ${ }^{36}$ nel quale s'incunea l'apposizione 'parentetica' o 'interposta'. ${ }^{37}$ Questa raffinata costruzione del verso non è un mero esercizio di stile, ma è in funzione del senso o meglio è la forma dell'espressione del dolore. All'effetto della figura stilistica dell'iperbato, che sospende, enfatizzandolo, il possessivo meae, s'aggiunge quello non solo del metro - l'aggettivo è messo in rilievo prima di tritemimera -, ma anche della sintassi, in quanto in latino la collocazione prenominale del possessivo è, come è noto, marcata. La pausa metrica e la sintassi spezzata, per via dell'apposizione interposta felix quondam pecus, sembrano inoltre avere un valore mimetico, suggeriscono, nelle parole di Melibeo, un sospiro, una pausa piena di pathos seguita da una dolorosa riflessione: il pastore, nel rivolgersi alle sue caprette, indugia per un momento a ricordare un tempo ormai perduto nel quale sono state felici. ${ }^{38} \mathrm{~A}$ ciò si aggiunga che l'apposizione in Virgilio ha spesso, come qui, un valore patetico, ${ }^{39}$ e che in particolare quondam ha una nuance patetica quando evoca un passato felice che non tornerà. ${ }^{40} \mathrm{E}$ in questo contesto che va misurata l'espressività del sintagma non ego uos, una formula che dice qui il doloroso commiato dalla vita di un tempo. Nella negazione, evidenziata dall'amplissimo iperbato (non ... uidebo) e dalla triplice anafora in variatio (non ego, carmina nulla, non me), è tutto il dramma 
dell'assenza: le sue caprette continueranno a pascolare, ma altrove; Melibeo non intonerà più i suoi canti e non potrà più ammirarle mentre s'inerpicano sulle rupi. ${ }^{41}$

Un altro passo virgiliano nel quale lo stilema non ego te è variato, ma presenta ancora la medesima espressività dei passi precedentemente citati è Aen.10.789 ss.

ingemuit cari grauiter genitoris amore, ut uidit, Lausus, lacrimaeque per ora uolutae.

hic mortis durae casum tuaque optima facta,

si qua fidem tanto est operi latura uetustas,

non equidem nec te, iuuenis memorande, silebo.

('Profondamente gemette per la morte del suo caro padre, come lo vide, Lauso, e lagrime gli scorsero lungo le gote. Qui la vicenda della tua triste morte e il tuo nobile gesto, se di quest'alta impresa farà fede in futuro l'antica memoria, io certo non lascerò nel silenzio, né te, giovinetto degnissimo.')

dove il nesso non equidem, equivalente e persino più espressivo di non ego ('non io certo'), è impiegato in un passo altamente patetico e ancora in convergenza con apostrofe e litote. Il poeta interrompe la narrazione per rivolgersi al giovane Lauso, che, destinato di lì a poco a soccombere per mano di Enea (dopo aver difeso con memorabile gesto di pietà filiale il padre Mezenzio), vivrà, o almeno questa è la speranza di Virgilio, per sempre nella poesia dell'Eneide.$^{42}$ L'espressività patetica della dizione è evidente, oltre che nel nesso si qua, che introduce una speranza piena di pathos (in questo caso, che il tempo non cancelli la gloria delle imprese), ${ }^{43}$ anche nel termine iuuenis, che sottolinea la giovane età del guerriero destinato ad una mors immatura - è il tipo di pathos che Macrobio (Sat.4.3) definisce appunto ab aetate -, e nel termine solenne memorande, efficacemente giustapposto al quasi sacrale silebo (selezionato da Virgilio al posto dell'isoprosodico tacebo) ${ }^{44}$ a significare che la memoria va eternata nella parola, non nel silenzio.

20 L'autorità di Virgilio sancisce la definitiva nobilitazione letteraria di questo nesso, che, ormai a tutti gli effetti stilema, diviene possesso della poesia augustea. Orazio, ad esempio, impiega la sequenza non ego te 4 volte, sempre ad inizio di frase, ma riservandola ai metri lirici delle $0 \mathrm{di}^{45}$ Se le due occorrenze nel primo libro sembrano meno significative, ${ }^{46}$ l'influsso di Virgilio è evidente nei due casi tratti dal quarto libro. In 4.9.29 ss.

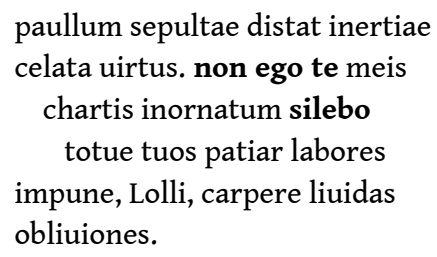

('Tra il valore nell'ombra e la viltà sepolta, la distanza non è grande. Ma non te, Lollio, io non sopporterò che le mie carte tacciano di te, non ti rendano onore, che l'oblio maligno rubi tante opere tue senza vendetta.')

21 i punti di contatto con il testo di Virgilio appaiono evidenti sia a livello di strutture sia di motivi. Dopo un catalogo di poeti ed eroi resi immortali dalla poesia, il poeta si 
rivolge al console Lollio, assicurandogli un posto nei suoi versi, per strapparlo all'oblio, triste destino per tanti uomini che vissero con valore, ma che non ebbero la fortuna di essere cantati dai poeti. Qui la sequenza non ego te, rafforzata dal possessivo meis, in fine di verso e staccato dal suo sostantivo in enjambement, ${ }^{47}$ richiama l'uso virgiliano sia per la litote panegiristica sia per l'impiego del solenne silebo. Mi sembra evidente che ci troviamo di fronte ad una sorta di 'ironia metaletteraria'. Il tono dell'encomio è stato, infatti, ritenuto da molti, e probabilmente a ragione, ironico, in quanto i meriti e le virtù del destinatario dell'ode sono piuttosto discutibili: se l'opus di Lauso è magnum e merita di essere eternato dalla poesia, non si può dire lo stesso per le imprese del console Lollio, che fu responsabile della cosiddetta clades Lolliana (17 o 16 a.C.), durante la quale fu sottratta l'aquila della $\mathrm{V}$ Legio. ${ }^{48}$

22 La virgilianità della sequenza non può che essere confermata, inoltre, dall'occorrenza nella dodicesima ode del quarto libro (4.12.21 ss.), un'ode che, dedicata a Virgilio, ${ }^{49}$ è intrisa, nella lingua e nello stile, di virgilianismi che richiamano soprattutto le Bucoliche e le Georgiche: ${ }^{50}$

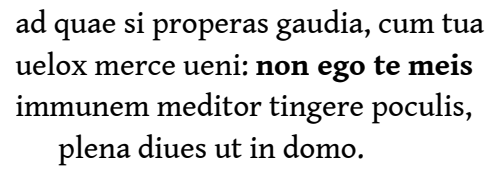

('Se hai fretta di queste gioie vieni svelto, con la tua mercanzia: io non intendo darti da bere senza contributo, come in casa del ricco che ha ogni cosa.')

Il tono è ironico, scherzoso. Orazio, pur non avendo nulla da offrirgli, invita l'amico a bere da lui: sarà lo stesso poeta mantovano a dover portare la sua 'merce', il nardo, con cui guadagnarsi il vino.

Questi casi appena commentati mostrano come i poeti prendano consapevolezza del valore stilistico di questo stilema impiegandolo con precise finalità espressive.

Che il nesso non sia impiegato in egual misura dai vari autori è, inoltre, conferma del fatto che non si tratta di una sequenza casuale di parole, ma che al contrario è impiegato deliberatamente. Non deve meravigliare, ad esempio, che non ego te non sia presente nel lungo poema lucreziano, nell'epos di Lucano o in Properzio, mentre registra ben tre occorrenze (una con numquam in luogo di non) nella breve Ciris (541 esametri), un'opera - è questa una delle ipotesi più plausibili - che è da considerarsi come un falso intenzionale, databile probabilmente nel I o II sec. d.c. (più difficilmente nel III sec. d.C.)..$^{51}$ Quest'ultimo testo è particolarmente interessante in quanto l'autore, nell'impersonare' Virgilio, ricorre, oltre a emistichi o a interi versi virgiliani, anche alla formula non ego te, per virgilianizzare ulteriormente il suo testo. Al verso 18 non ego te talem uenerarer munere tali ('io non onorerei la tua egregia persona con un dono siffatto'), ${ }^{52}$ all'interno di un lungo proemio, la sequenza non ego te è chiaramente virgiliana, impiegata com'è in un'apostrofe panegiristica, rivolta ad un Messalla. ${ }^{53}$ L'anonimo autore, impersonando un Virgilio che si è da poco dato alla filosofia epicurea, si giustifica per aver deciso di riprendere un poemetto giovanile, la Ciris appunto, rinunciando ad un poema filosofico, che sarebbe stato per lui troppo impegnativo e prematuro.

La Ciris, seppur caratterizzata da un livello stilistico disomogeneo, presenta alcuni passaggi stilisticamente ricercati e caratterizzati da un profondo pathos. È così, ad 
esempio, nelle parole della nutrice di Scilla, Carme, la quale in una commossa apostrofe si rivolge alla figlia Britomarti, suicidatasi per scampare alla violenza di Minosse, del quale è ora tragicamente innamorata la sua giovane protetta (vv.307 ss.):

numquam ego te summo uolitantem uertice ...

Hyrcanos inter comites agmenque ferarum

conspiciam nec te redeuntem amplexa tenebo. ${ }^{54}$

('mai più ti vedrò correre sull'altissima cima di un monte [...]

accompagnata dai cani d'Ircània e tra branche di fiere

né al tuo ritorno ti terrò fra le braccia.')

Mi sembra evidente che nella sequenza, con la leggera variatio della negazione numquam in luogo di non e con il verbum videndi (conspiciam) in rilievo in enjambement, l'anonimo poeta abbia voluto richiamare il citato modello catulliano dell'apostrofe al fratello, mediante il modulo patetico del 'non ti vedrò mai più' (lì una disperata domanda, qui una dolorosa constatazione). La sequenza non ego te torna, sempre nelle parole di Carme, al verso 328

non ego te incepto, fieri quod non pote, conor

flectere amore, nec est cum dis contendere nostrum,

sed patris incolumi potius denubere regno

atque aliquos tamen esse uelis tibi, alumna, penates

('Non io tento di distoglierti dall'amore che provi - questo non è possibile, né è mia intenzione contendere con gli dèi ma spero piuttosto che tu voglia le nozze, sano e salvo il regno di tuo padre, e tuttavia qualche focolare domestico.')

ma con diversa enfasi (si tratta di aversio): la nutrice dichiara di non voler distogliere Scilla dall'amore - al quale è impossibile opporsi -, ma la invita a trovarsi un altro marito che non sia Minosse, l'uomo che sta assediando il regno del padre.

Tornando alla poesia augustea, la sequenza registra tre occorrenze in Tibullo, tutte ad inizio di esametro ${ }^{55}$ e nella medesima composizione, la sesta elegia del primo libro. Particolarmente significativo è Tib.1.6.29 s.

non ego te laesi prudens (ignosce fatenti): iussit Amor: contra quis ferat arma deos?

('Non ti ho offeso a cuor leggero: perdona a chi confessa; Amore me l'ha imposto. Chi combatterebbe contro gli dei?) ${ }^{56}$

dove il poeta chiede, paradossalmente e non senza toni ironici, al marito della sua Delia di controllarla, per evitare che possa vedere un suo nuovo amante, e si giustifica per averla posseduta in passato asserendo di averlo fatto senza volerlo, quasi costretto da Amore. Il passo presenta la litote giustificatoria non prudens, che rientra nel modulo retorico della concessio per purgationem - la richiesta di perdono per aver commesso un misfatto per necessitudo, fortuna o imprudentia $-^{57}$ con un participio che richiama il citato Amph.531 Non ego te hic lubens relinquo neque abeo abs te, un verso non privo, come si è detto, di sfumature ironiche.

Tibullo ripropone la sequenza al verso 57 sempre della sesta elegia del primo libro:

non ego te propter parco tibi, sed tua mater me mouet atque iras aurea uincit anus.

haec mihi te adducit tenebris multoque timores coniungit nostras clam taciturna manus, 
('No, non è per te che mi impietosisco:

mi commuove tua madre, quella vecchia d'oro disarma la mia collera;

lei nelle tenebre a me ti conduce e piena di paura, di nascosto,

ci congiunge senza una parola le mani')

Qui, però, il sintagma è diverso dal punto di vista del significato (recuperato comunque dall'espressione parco tibi) in quanto il pronome te è retto dalla preposizione propter, la cui anastrofe rende possibile la sequenza. Il poeta vuol mantenere la giustapposizione pronominale ego te per legarla al distico successivo, dove si ripete variata in mihi te, un accostamento, quest'ultimo, che è chiara icona del contatto, come è specificato dall'espressione coniungit nostras ... manus. Anche nell'ultimo caso tibulliano (1.6.73 ss.)

non ego te pulsare uelim, sed, uenerit iste

si furor, optarim non habuisse manus

nec saeuo sis casta metu, sed mente fideli: mutuus absenti te mihi seruet amor.

('Non vorrei mai percuoterti, ma se un tale furore m'assalisse, il desiderio sarebbe di non avere mani. Non essere però casta per timore di pene:

l'amore a tua volta ti conservi fedele anche quando sono lontano')

la sequenza è motivata dall'accostamento pronominale (te mihi) del distico successivo.

Con Tibullo, la formula, ormai consolidato stilema esametrico, transita nel mondo elegiaco e viene adottata, con una certa frequenza, anche da Ovidio ( 7 occorrenze). ${ }^{58} \mathrm{Va}$ tuttavia considerata l'inclinazione del poeta di Sulmona all'accostamento pronominale ego te (27 occorrenze), che è particolarmente frequente nelle Heroides (12 casi), per via del carattere epistolare (che implica la presenza del pronome di prima e seconda persona) e amoroso (ego te rappresenta iconicamente in questi casi la vicinanza dei due amanti) della composizione. Ovidio fa un uso cosciente del nesso, riconoscendone, ad esempio, il carattere panegiristico, riproposto, non a caso, in Fasti $3.55 \mathrm{~s}$.

non ego te, tantae nutrix Larentia gentis, nec taceam uestras, Faustule pauper, opes

('Non io te tacerò, Larenzia, nutrice di così nobile stirpe, né la vostra assistenza, o umile Faustolo.' Trad. M. Fucecchi)

Qui il distico ricalca il modello virgiliano, oltre che per la litote panegiristica, anche per la citazione di due nomi (Larentia, Faustule), per l'enjambement della forma verbale e per la collocazione 'artistica' del vocativo, incorniciato tra aggettivo e sostantivo (uestras, Faustule pauper, opes).

Più catulliani, invece, nel loro carattere 'elegiaco', sono i casi tratti dalle Heroides, come è naturale conseguenza delle diverse atmosfere e registri linguistici. In epist.10.67 s.

non ego te, Crete centum digesta per urbes, aspiciam, puero cognita terra Ioui.

('No, io non ti vedrò più, o Creta divisa in cento città, terra conosciuta da Giove bambino!') $)^{59}$

il sofferto commiato di Arianna dalla terra amata, con il motivo del 'non ti rivedrò più' ricorda il modello sia catulliano, del quale riprende anche l'enjambement della forma verbale (65.10 s. numquam ego te ... / aspiciam posthac), sia virgiliano (b.1.75 s. non ego uos posthac ... /... uidebo). 

esempio tratto dalle Heroides (12.195 s.) è l'implorazione piena di pathos di Medea, che chiede al suo Giasone non di compiere imprese straordinarie, ma semplicemente di ricambiare il suo amore:

non ego te imploro contra taurosque uirosque, utque tua serpens uicta quiescat ope,

('Io non faccio appello a te contro tori o uomini, né perché un drago dorma domato dal tuo intervento')

Nella poesia flavia il nesso sarà utilizzato, invece, solo tre volte (una occorrenza rispettivamente in Valerio Flacco, Silio Italico e Stazio) senza il valore panegiristico, ma in ogni caso in contesti particolarmente patetici.

In Val.Flac.8.92 ss.

ipsa caput cari postquam Medea draconis uidit humi fusis circum proiecta lacertis seque suumque simul fleuit crudelis alumnum. "non ego te sera talem sub nocte uidebam sacra ferens epulasque tibi, nec talis hianti mella dabam ac nostris nutribam fida uenenis. quam grauida nunc mole iaces, quam segnis inertem flatus habet! nec te saltem, miserande, peremi.

('Medea, quando vede atterrato il serpente, si getta per stringerlo tra le sue braccia; si è fatta crudele, ma piange per se stessa e per lui: «No, nella notte profonda non ti vedevo così quando a te portavo le offerte consacrate ed il cibo; io non ero così quando tu venivi affamato e ti davo il miele e, fidata, ti nutrivo con i miei filtri. Come pesante, adesso, ed immobile giaci! Respiri inerte con esile fiato. Se almeno ti avessi fatto morire, infelice!'. Trad. F. Caviglia)

ancora un patetico commiato. Medea, per aiutare Giasone nella sua impresa, addormenta il guardiano del vello d'oro, quel serpente che era stato anche un suo caro, inseparabile amico. La donna, alla vista della bestia addormentata, è presa quasi da un rimorso per l'inganno ai danni dell'animale, e rievoca il tempo passato, quando gli portava fedelmente il suo cibo incantato.

In Stazio Theb.11.341 ss.

haec tibi canities, haec sunt calcanda, nefande, ubera, perque uterum sonipes hic matris agendus. parce: quid oppositam capulo parmaque repellis? non ego te contra Stygiis feralia sanxi uota deis, caeco nec Erinyas ore rogaui.

('Devi calpestare, maledetto, questa canizie, questo seno, attraverso il ventre della madre devi spingere questo cavallo. Ma fermati: perché mi respingi con l'elsa della spada e con lo scudo? Io non ho rivolto contro di te lugubri voti agli dèi dello Stige, né ho invocato le Erinni con il volto cieco.' Trad. A. Traglia, G. Aricò) 
43 in un altro passo altamente patetico - Giocasta supplica Eteocle di deporre le armi - il sintagma, impiegato com'è nella figura dell'aversio,${ }^{60}$ dice la disperata giustificazione della madre: non lei, ma solo Edipo ha invocato la maledizione contro il figlio.

44 In Silio Italico (7.38) il nesso torna in un passo chiaramente ispirato a Virgilio, nelle fiere parole dell'eroe aretino Cilnio, quando provocatoriamente si accinge non ad un'apostrofe panegiristica ma a narrare brevemente al nemico Annibale, che lo tiene prigioniero, le imprese dei Fabii per evidenziare il valore del Cunctator: non ego te longa serie per singula ducam / hoc sat erit; nosces Fabios certamine ab uno ("Non starò a dirti nel dettaglio la lunga successione dei fatti. Questo ti basterà; da una sola battaglia comprenderai chi sono i Fabii.' Trad. M. A. Vinchesi). ${ }^{61}$

45 Questa catena di citazioni, che, a mio avviso, dimostra chiaramente la letterarietà del sintagma, è suggellata da un passo di Ausonio, un fine poeta che, per la sua formazione di rhetor e grammaticus, ha una profonda conoscenza dei classici e in particolare di Virgilio, il suo principale modello stilistico e formale. ${ }^{62}$ In Parent. $10.1 \mathrm{~s}$.

Non ego te infletum memori fraudabo querella, primus, nate, meo nomine dicte puer

'Io non priverò te, non ancora pianto, di lamenti che ti ricordino, o figlio chiamato per primo con il mio nome' (Trad. M. Lolli)

il poeta dinanzi al dramma della perdita del caro figlioletto, un ớwpoৎ tragicamente scomparso in tenerissima età, ne affida la memoria al potere della poesia e nel far ciò ricorre alla litote panegiristica (non ego te ... memori fraudabo querella), che nella sua memoria di lettore innesca un complesso e raffinato gioco di citazioni. Catullo, Orazio e soprattutto Virgilio ${ }^{63}$ riecheggiano nella sua poesia, ma quella che nei poeti è semplice finzione letteraria, elegante gesto di citazione, diviene per Ausonio un disperato, drammatico tentativo di affrontare la vita ed esorcizzare il dolore.

Alla fine della nostra discussione appare chiara la storia di questo sintagma essenzialmente poetico (è quasi del tutto assente in prosa), che, formato a partire dalla giustapposizione pronominale ego-te, è impiegato in contesti emotivi. Non attestata in Terenzio, ma ampiamente sfruttata in Plauto - ciò è prova che non sia semplicemente ascrivibile alla lingua d'uso -, la sequenza non ego te si configura come un vero e proprio stilema poetico, impiegata com'è costantemente ad inizio di verso (quasi sempre di esametro), in domande retoriche, formule di giustificazione (aversio) o di commiato. Il passaggio e la nobilitazione alla poesia alta si deve a Catullo, che, riprendendo un passo dell'Amphitruo pieno di ironia e comicità (Giove si congeda dall'insaziabile Alcmena dopo una notte d'amore), lo carica di memorabile espressività patetica nello struggente commiato di Egeo nei confronti del figlio (64.222), mentre lo ripropone, variato con la negazione numquam e associato al tema patetico del 'non ti rivedrò più', nella commossa allocuzione al fratello defunto $(65.10$ s.). Dopo Catullo, il sintagma, ormai stilema, è impiegato da Virgilio, che lo adatta ai diversi registri stilistici delle sue opere, innovandolo ulteriormente: se, infatti, nelle Bucoliche l'uso ricalca quello delle interrogative plautine, nelle Georgiche, come si è visto, la sequenza non ego te è impiegata in un'apostrofe panegiristica associata alla litote, uno stilema qui ironicamente alto, diretto com'è ad una pianta, ma nell'occorrenza dell'Eneide realmente patetico, nell'apostrofe panegiristica a due guerrieri (Aen.10.185). Per Orazio (4 occorrenze) lo stilema ha ormai un sapore virgiliano, se lo impiega ancora in un'apostrofe panegiristica (c.4.9.30-1) e addirittura in un' un'allocuzione allo stesso 
Virgilio (4.12.22). L'assenza del sintagma in alcuni autori (Lucrezio, Lucano e Properzio) conferma che il nesso non è una sequenza casuale. Impiegato tre volte in una sola elegia di Tibullo (la sesta del primo libro), sette volte in Ovidio e una volta in Valerio Flacco, Stazio e Silio, e sempre in contesti comparabili, il sintagma registra un'ultima e isolata attestazione in Ausonio, che combina, come si è visto, il modello catulliano e quello virgiliano in un passo di struggente pathos.

\section{BIBLIOGRAFIA}

Agnesini 2004: A. Agnesini, Plauto in Catullo, Bologna.

Augello 1977: G. Augello, Studi apuleiani. Problemi di testo e loci vexati delle Metamorfosi, Palermo. Bellandi 2007: F. Bellandi, Lepos e Pathos. Studi su Catullo, Bologna.

Bellincioni 1985: M. Bellincioni, Felix/infelix, in Enciclopedia Virgiliana, II, a cura di F. Della Corte, Roma, pp.486-488.

Christenson 2000: D. Christenson, Plautus: Amphitruo, Cambridge.

Clausen 1994: W. Clausen, A Commentary on Virgil Eclogues, Oxford.

Conte 2007: G. B. Conte, The Poetry of Pathos. Studies in Virgilian Epic, ed. S.J. Harrison, Oxford 2007.

Cucchiarelli 2012: A. Cucchiarelli, Publio Virgilio Marone, Le Bucoliche. Introduzione e commento, Roma.

Dainotti 2015: P. Dainotti, Word Order and Expressiveness in the Aeneid, Berlin/Boston.

Fasce 1987: S. Fasce, Nascor, in Enciclopedia Virgiliana, III, a cura di F. Della Corte, Roma, pp. 664-664.

Fedeli 2019 : P. Fedeli, Orazio, Carm. 4, 12. L'importanza di chiamarsi Virgilio, «Atene e Roma» 13, pp. $1-12$.

Fernandelli 2012: M. Fernandelli, Via Latina : Studi su Virgilio e sulla sua fortuna, Trieste.

Filoche 2007: C. Filoche, Les comiques dans l'intertexte des Bucoliques, in C. Filoche (ed.), L'intertexte virgilien et sa réception: écriture, récriture et réflexivité chez Virgile et Rutilius Namatianus, Dijon, pp. 55-85.

Fordyce 1961: C. J. Fordyce, Catullus. A Commentary, Oxford.

Gagliardi 2017: P. Gagliardi, Tua cura Lycoris: lessico erotico e schema Cornelianum da Virgilio agli elegiaci nel segno di Gallo, «Wiener Studien» 130, 2017, pp.183-200.

Hardie 1994: P. Hardie, Virgil, Aeneid Book IX, Cambridge.

Harrison 1991: S. J. Harrison, Vergil Aeneid 10. With Introduction, Translation, and Commentary, Oxford.

Harrison, Frangoulidis, Papanghelis 2018: S. J. Harrison, S. Frangoulidis, T. D. Papanghelis (eds.), Intratextuality and Latin Literature, Berlin/Boston. 
Helm 1931³: R. Helm, Apulei Platonici Madaurensis Metamorphoseon libri XI, Editio tertia, Lipsiae $\left(1907^{1}\right)$.

Heuzé 1985: P. Heuzé, L'image du corps dans l'oeuvre de Virgile, Roma.

Hofmann, Szantyr (Traina) 2002: J. B. Hofmann, A. Szantyr, Stilistica latina, a cura di A. Traina, Bologna.

Hofmann (Ricottilli) $2003^{3}$ J. B. Hofmann, La lingua d'uso latina. Introduzione, traduzione e note a cura di Licinia Ricottilli. Terza edizione aggiornata, Bologna $\left(1980^{1}\right)$.

Horsfall 2000: N. Horsfall, Virgil, Aeneid 7: A Commentary, Leiden/Boston.

Horsfall 2003: N. Horsfall, Virgil, Aeneid 11: A Commentary, Leiden/Boston.

Horsfall 2006: N. Horsfall, Virgil, Aeneid 3: A Commentary, Leiden/Boston.

Horsfall 2013: N. Horsfall, Virgil, Aeneid 6: A Commentary, Berlin/Boston.

Kroll 19897: W. Kroll, Catull, Stuttgart (Breslau 1922¹).

Kroll (Lunelli) 1988³ W. Kroll, La lingua poetica romana, in A. Lunelli (a cura di), La lingua poetica latina, con saggi di W. Kroll, H. H. Janssen, M. Leumann, Bologna (1974¹), pp.1-66.

Landolfi 2011: L. Landolfi, Porcii Licini ep. 6, Bl. Un nuovo caso di apposizione parentetica?, «Rivista di filologia e di istruzione classica» 139 (2), pp.310-317.

La Penna 1989: A. La Penna, Cum flore, Maecenas, rosarum: su una collocazione artistica del vocativo in poesia latina, in G. G. Biondi (ed.), Mnemosynum, Studi in onore di A. Ghiselli, Bologna, pp.335-353.

La Penna 2013: A. La Penna, La letteratura latina del primo periodo augusteo (42-15 a.C.), Roma/Bari.

Lausberg 1960: H. Lausberg, Handbuch der literarischen Rhetorik, Monaco.

Lee 1979: M. O. Lee, Fathers and Sons in the Aeneid: Tum Genitor Natum, Albany.

Lenchantin de Gubernatis 1928: M. Lenchantin de Gubernatis, Il libro di Catullo, Torino.

Lolli 1997: M. Lolli, D. M. Ausonius, Parentalia. Introduzione, testo, traduzione e commento, Bruxelles.

Maltby 2002: R. Maltby, Tibullus: Elegies. Text, Introduction and Commentary, Cambridge. Marouzeau 1946²: J. Marouzeau, Traité de stylistique latine. Paris (1935¹).

Maurach 1983: G. Maurach, Enchiridion poeticum. Hilfsbuch zur lateinischen Dichtersprachen, Darmstadt 1983.

Maurach 2006²: G. Maurach, Lateinische Dichtersprache, Darmastadt (1995¹).

Moskalew 1982: W. Moskalew, Formular Language and Poetic Design in the Aeneid, Leiden.

Munzi 1985: L. Munzi, Quondam, in Enciclopedia Virgiliana, IV, a cura di F. Della Corte, Roma, pp. 384-385.

Mynors 1990: R. A. B. Mynors, Virgil, Georgics, Oxford.

Nisbet 2008: R. G. M. Nisbet, The Style of Virgil's Eclogues, in K. Volk (ed.), Oxford Readings in Classical Studies, Vergil's Eclogues, Oxford, pp.48-63.

Nisbet,

Hubbard 1970: R. G. M. Nisbet, M. Hubbard, A Commentary on Horace Odes, Book I, Oxford. Nuzzo 2003: G. Nuzzo, Gaio Valerio Catullo, Epithalamium Thetidis et Pelei (c. LXIV), Palermo. 
O'Daly 2004: G. O' Daly, Sunt etiam Musis sua ludicra: Vergil in Ausonius, in Romane memento: Vergil in the Fourth Century, edited by R. Rees, London, pp.141-154.

Pascucci 1987: G. Pascucci, Negazioni, in Enciclopedia Virgiliana, III, a cura di F. Della Corte, Roma, pp.678-682.

Pease 1935: S. A. Pease, Publi Vergili Maronis Aeneidos liber quartus. Cambridge Massachusetts.

Peirano 2014: I. Peirano, Appendix Vergiliana, in The Virgil Encyclopedia, ed. Richard F. Thomas and Jan M. Ziolkowski (Chichester/Malden, MA), vol. I, pp.104-111.

Perutelli 1979: A. Perutelli, La narrazione commentata. Studi sull'epillio latino, Pisa.

Prete 1984: S. Prete, Ausonio, in Enciclopedia Virgiliana, I, a cura di F. Della Corte, Roma, pp.422-423. Ricottilli 1990: L. Ricottilli, Taceo, in Enciclopedia Virgiliana, V*, a cura di F. Della Corte, Roma, pp. 7-14.

Ricottilli 2000: L. Ricottilli, Gesto e parola nell'Eneide, Bologna.

Sharrock, Morales 2001: A. Sharrock, H. Morales (eds.), Intratextuality: Greek and Roman Textual Relations, Oxford.

Skutsch 1956: O. Skutsch, Zu Vergils Eklogen, «Rheinisches Museum für Philologie» 99, pp.193-201. Slusher 1930: M. F. Slusher, The Use of Litotes in Vergil's Aeneid, «Transactions and Proceedings of the American Philological Association» 61, p.XXXV.

Smith 1913: K. F. Smith, The Elegies of Albius Tibullus. The Corpus Tibullianum. Edited with Introduction and Notes on Books I, II and IV 2-14, New York.

Solodow 1986: J. B. Solodow, Raucae, tua cura, palumbes: Study of a Poetic Word Order, «Harvard Studies in Classical Philology» 90, pp.129-153.

Tarrant 2012: R. J. Tarrant, Virgil, Aeneid, Book XII, Cambridge.

Thomas 1988: R. F. Thomas, Virgil. Georgics. Volume 1, Books I-II, Cambridge.

Thomas 2011: R. F. Thomas, Horace: Odes Book IV and Carmen Saeculare, Cambridge.

Timpanaro 1978: S. Timpanaro, Contributi di filologia e di storia della lingua latina, Roma.

Traina 1989: A. Traina, Note virgiliane. 1. Un modulo ritmico-sintattico: epos e pathos, in A. Traina, Poeti latini e (neolatini). Note e saggi filologici, III, Bologna, pp.133-137 (=AA.VV. Kontinuität und Wandel. Festschrift Munari, Hildesheim 1986, pp.35-42).

Traina 1994: A. Traina, Bilancio di un'enciclopedia, in Poeti latini (e neolatini). Note e saggi filologici, Bologna 1994, pp.123-137 (=«Rivista di filologia e di istruzione classica» 120, 1992, pp.111-123).

Traina 1998: A. Traina, Un probabile verso di Ennio e l'apposizione parentetica, in Poeti latini (e neolatini). Note e saggi filologici, Bologna 1998, pp.11-17 (=«Materiali e discussioni per l'analisi dei testi classici» 34, 1995, pp.187-193).

Traina 20042: A. Traina, Virgilio. L'utopia e la storia. Il libro XII dell'Eneide e antologia delle opere. Edizione riveduta, aggiornata e ampliata, Torino $\left(1997^{1}\right)$.

Venini 1970: P. Venini, P. Papini Stati Thebaidos liber undecimus. Introduzione, testo critico, commento e traduzione, Firenze.

Zucchelli 1985: B. Zucchelli, Diminutivi, in Enciclopedia Virgiliana, II, a cura di F. Della Corte, Roma, pp.75-77. 


\section{NOTE}

1. Ringrazio i referees anomini di Dictynna e i professori Stefano Grazzini, Stephen Harrison e Alexandre Pinheiro Hasegawa per l'attenta lettura e i preziosi suggerimenti, che hanno notevolmente migliorato questo lavoro.

2. Non si sono considerate, ovviamente, le occorrenze (12) in scoliasti e commentatori. Il sintagma è impiegato una sola volta da Cicerone, in un contesto particolarmente patetico, in ad Quint.1.3.1 Mi frater, mi frater, mi frater, tune id veritus es ne ego iracundia aliqua adductus pueros ad te sine litteris miserim? aut etiam ne te videre noluerim? Ego tibi irascerer? tibi ego possem irasci? Scilicet, tu enim me adflixisti; tui me inimici, tua me invidia ac non ego te misere perdidi ('Fratello mio, fratello mio, fratello mio carissimo, e come hai potuto temere che per un moto qualsiasi di collera io ti abbia mandato schiavi senza una lettera per te, che per di più io abbia evitato di incontrarmi teco? Proprio io essere in collera con te, proprio con te io potrei adirarmi? Oh, davvero sei tu che mi hai portato alla rovina, davvero me i tuoi nemici, me il tuo odio, e non invece io te ho travolto miseramente nella catastrofe!'. Trad. C. Vitali), un passo dove l'espressività della sequenza converge con l'enfasi dell'accostamento di pronomi e/o aggettivi possessivi di prima e seconda persona (ego tibi; tibi ego; tui me; tua me); e ancora da Apuleio, un autore che, come è noto, non disdegna poetismi (vedi Kroll (Lunelli) $1988^{3}$, p.23), in Met.8.12 Non ego <te> gladio, non ferro petam; absit, ut simili mortis genere cum marito meo coaequeris: vivo tibi morientur oculi nec quiquam videbis nisi dormiens ('Non ti assalirò con la spada né con altro tipo di fendente; che non accada che con una simile morte tu possa essere uguale a mio marito: a te vivo moriranno gli occhi e non vedrai più nulla, se non dormendo'), dove la sequenza non ego te gladio (il pronome te è, però, frutto di un'integrazione di Helm $1931^{3}$, ad loc., poi scartata dallo stesso Helm nella successiva ristampa con correzioni. Vedi Augello 1977, p.182 con bibliografia) avrebbe, a mio avviso, anche il vantaggio di conferire alla frase un ritmo perfettamente esametrico (equivale ad un emistichio fino alla pentemimera), che qui si addice alla scena di alto pathos: nelle parole di Carite, che si appresta ad assalire Trasillo (l'assassino di suo marito), la rassicurazione appare ironica (è un caso di oppositio in imitando rispetto agli esempi plautini): può dormire tranquillo, perché non sarà assalito con la spada,ma gli saranno solo cavati gli occhi.

3. Plaut. Amph.518, 531; Capt.564; Curc.713; Men.407, 511, 632; Poen.429, 1040; Rud.1125; Truc.606; frg.137; Afran. 253 R. Le traduzioni dei passi plautini sono di E. Paratore, quelle dell'Eneide di R. Scarcia, le traduzioni delle altre citazioni, se non diversamente indicato, sono le mie.

4. La giustapposizione pronominale, un tratto sia della lingua popolare sia alta (vedi Horsfall 2000, ad Aen.7.427, con bibliografia), ha, nel caso di ego te, la funzione di sottolineare, non senza un'intonazione 'affettiva', il rapporto tra chi parla e chi ascolta. Si veda Ricottilli 2000, p.90, Hofmann (Ricottilli) 2003³, p.240, Thomas 2011, ad Hor.C.4.12.22, e, per esempi di giustapposizione di pronomi (e di pronomi e aggettivi possessivi) in Virgilio, Dainotti 2015, pp.237 s.

5. Su non ego a inizio di esametro vedi Pascucci 1987, p.679.

6. In Rud.1125, invece, la domanda retorica suona come una minaccia: non ego te comprimere possum sine malo? ('Insomma, l'orifizio non te lo posso turare se non ti scarico addosso una bella sonata?'). In altre due occorrenze plautine il nesso è impiegato invece in una sorta di affermazione denigratoria, come in Curc.713 Non ego te flocci facio: ne me territes, un insulto intimidatorio ('Io neanche ti vedo, non credere di farmi paura') che ritorna anche in Truc.606 non ego te flocci facio ('A me di te non me ne frega niente').

7. Sulla comicità di questa situazione vedi Christenson 2000, ad loc. («Jupiter [...] is comically trying to escape his sexually insatiable mistress»).

8. Quint.Inst.9.2.39 Sed illa quoque uocatur auersio quae a proposita quaestione abducit audientem ('Ma viene chiamata apostrofe anche la figura che distoglie l'uditorio dall'argomento proposto'. Trad. M. Calcante). Per alcuni esempi della figura si veda Pease 1935, ad Aen.4.425. Può essere considerato un caso di aversio anche Pseud. 1040 s. non ego te ad illum duco dentatum uirum / 
Macedoniensem, qui te nunc flentem facit ('No, non ti conduco dall'orco con le zanne, da quel Macedone della malora che ti sta facendo piangere tanto').

9. Non è rilevata nei principali commenti (ma Nuzzo 2003, ad loc. considera comunque non ego te uno stilema incipitario) né in Agnesini 2004, uno studio specifico sulla presenza di Plauto in Catullo.

10. Su questo tipo di pathos si veda Horsfall 2003, ad Aen.11.42 (con bibliografia).

11. Sulla centralità del rapporto padre-figlio nell'Eneide si veda Lee 1979. Una vita lunga non è, nemmeno per un uomo che, come Egeo, è vicino alla sua fine, preferibile alla presenza del caro, unico figlio. In tal senso la lezione dei poziori longa è da preferirsi alla congettura longe di Hoeufft, non solo per la tendenza catulliana alla costruzione del verso con due sostantivi e due aggettivi (vedi Fordyce 1961, ad loc., che considera, quindi, l'aggettivo poco più che uno «stopgap»), ma anche e soprattutto per il senso. L'espressione longa vita, come nota Lenchantin

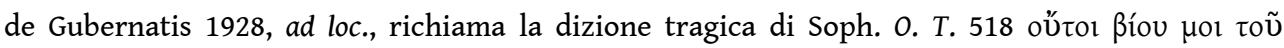

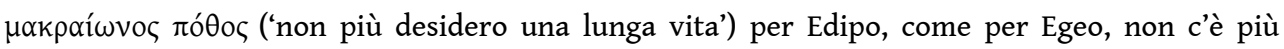
motivo di desiderare una lunga vita.

12. Sulla forma arcaica e poetica gnatus (in luogo di natus) si veda Horsfall 2013, ad Aen.6.868 (con bibliografia), sul valore affettivo di (g)natus, in particolare al vocativo e nelle apostrofi, Fasce 1987, pp.664 s. L'anafora di nate verrà ripresa da Virgilio in Aen.1.664 s. nate, meae uires, mea magna potentia, solus, / nate ('figliolo, mia sola energia, mia grande forza, / figliolo'), ma l'imitazione virgiliana più evidente è in Aen.5.724 s. nate, mihi uita quondam, dum uita manebat, / care magis, nate Iliacis exercite fatis ('Figliolo, della mia vita un tempo, finché vita mi restava, / più caro assai, figliolo messo a prova dai destini di Ilio'). Vedi Nuzzo 2003, ad Cat.64.215.

13. Sul pathos del pleonasmo extrema fine vedi Kroll $1989^{7}$, ad loc., su gaudens laetanti, Lenchantin de Gubernatis 1928, ad loc. Sul particolare tipo di pleonasmo costituito da sostantivo e aggettivo sinonimico vedi Hofmann-Szantyr (Traina) 2003, p.178.

14. Sul valore sentimentale di langueo e affini in Catullo vedi Fordyce 1961, ad loc. L'espressione languida lumina è, inoltre, un significativo richiamo intratestuale alle parole di Arianna (v. 188 languescent lumina): la giovane amante, come il vecchio Egeo, sono accomunati dal destino di morte provocato da Teseo. Vedi Nuzzo 2003, ad loc.

15. Su questa figura della lingua d'uso, particolarmente frequente nella lingua letteraria per il suo carattere enfatico e pregnante, si veda Hofmann (Ricottilli) $2003^{3}$, p.304, 308 ss. e 311, n.1, Hofmann-Szantyr (Traina) 2002, Index s.v. 'litote', Maurach 1983, pp.150 s. e 2006², pp.122 s. In mancanza di uno studio specifico sulla litote in Virgilio (Slusher 1930, XXXV è solo una breve sintesi di un intervento tenuto all'American Philological Association), si veda Pascucci 1987, p.679 (nella voce 'negazioni' discute alcune litoti costituite da non+avverbio o aggettivo).

16. L'immagine, già omerica (in Il.18.23 ss. Achille, nel piangere Patroclo, si cosparge il capo insanguinato di polvere), sarà ripresa da Virgilio in Aen.12.611 canitiem immundo perfusam puluere turpans ('imbrattando i bianchi capelli di polvere immonda') nella descrizione della reazione di Latino alla scoperta del suicidio di Amata e, con maggiore efficacia, in Aen.10.843 ss. agnouit longe gemitum praesaga mali mens: / canitiem multo deformat puluere et ambas / ad caelum tendit palmas et corpore inhaeret ('Riconobbe di lontano il compianto la mente presaga del male; / con molta polvere si scompiglia la canizie, e ambedue / le palme tende al cielo, e si avvinghia stretto al corpo'): Mezenzio, al pari di Egeo, presagisce nel suo cuore la sventura del figlio e s'abbandona ad un disperato dolore. Su questo gesto rituale del lutto si veda Harrison 1991, ad loc. e Heuzé 1985, p.488.

17. Propenderei qui per l'interpretazione secondo la quale la richiesta di Ortalo consisterebbe nella composizione di poesia originale e non nella traduzione, quest'ultima un surrogato con il quale Catullo vuole in parte accontentare l'amico. È evidente l'analogia con il carme 68A: anche lì una richiesta di poesia non può essere accolta a causa di un pathos impediente, anche lì una toccante apostrofe. Si veda Bellandi 2007, pp.38 ss. e 273. 
18. Bellandi 2007, p.275, n.636.

19. Agnesini 2004, p.12.

20. Tarrant 2012, ad Aen.12.310.

21. Si vedano le osservazioni di Clausen 1994, ad b.3.1, con utile elenco delle numerose reminiscenze plautine nell'ecloga.

22. Trad. di A. Traina come degli altri passi dalle Bucoliche.

23. Clausen 1994, ad loc.: «a Plautine vocative of objurgation [...] in non-dramatic poetry first here, and only here in V., then in Hor. Serm. 2.7.22, Pers. 2. 46». Il tono del passo è marcatamente plautino anche al verso 15 et si non aliqua nocuisses, mortuus esses ('e se in qualche maniera non gli facevi del male, eri morto'), dove si segnala il particolare uso, plautino e terenziano, di aliqua (con il sostantivo ratione sottinteso), ripreso solo qui in Virgilio, e l'iperbole colloquiale e plautina mortuus esses. Sulla presenza della commedia nelle Bucoliche si veda anche Filoche 2007, pp.55-85.

24. Trad. M. Ramous.

25. Su questa formula panegiristica si veda Nisbet, Hubbard 1970, ad Hor.C.1.12.21.

26. Su questa collocazione 'artistica' del vocativo in poesia latina si veda La Penna 1989, pp. 335-353.

27. Vedi Thomas 1988, ad loc: "with tumidis ('swelling') V. provides an elegant gloss on the etymology of bumastus, lit. 'with huge breasts' (bou- a prefix indicating large size, from ßoũ $\varsigma$, 'ox'; $\mu \alpha \sigma \tau o ́ c$, 'breast')».

28. Mynors 1990, ad loc.: «V. treats his grapes like individual captains in epic».

29. Su questa forma di auto-allusione, nota anche come 'orecchio interno', 'self-echo', 'ricorsività autoallusiva' o semplicemente 'intratestualità', si vedano i volumi a cura di Sharrock, Morales 2001 (sulla letteratura greca e latina) e Harrison, Frangoulidis, Papanghelis 2018 (sulla letteratura latina). Su Virgilio, oltre al volume di Moskalew 1982, si veda Dainotti 2015, Index, s.v. 'self-echo' (bibliografia a p.5, n.11). Non è da escludere che accanto al modello catulliano e plautino i due passi virgiliani in questione abbiamo come modello anche Ennio, un'ipotesi avanzata da Timpanaro 1978, pp.315 s.

30. Sulla convergenza di litote e apostrofe a sottolineare il tono panegiristico del passo vedi Harrison 1991, ad loc.

31. Nella poesia successiva, solo in Tib.2.4.16 e Stat.7.377. In Virgilio è sintomatico che non si registri nessuna occorrenza del sintagma non ego con gli altri pronomi personali.

32. Il parallelo, piuttosto evidente se si pensa al medesimo impiego dell'avverbio posthac, è segnalato da Kroll 19897 ad Cat.65.11.

33. Sull'importanza, in sede di analisi stilistica, della 'convergenza dei fattori stilistici', un concetto spizteriano noto anche come 'fascio di tratti stilistici', si vedano le osservazioni di Conte 2007, p.99. Qualcosa di simile (la convergenza di due o più figure a connotare lo stile patetico di Demostene) era stato già notato nella teoria antica, ad esempio, da Pseudo Longino, nel capitolo XX (dedicato appunto all'effetto di più figure) del suo trattato Sul sublime.

34. Su questo manierismo già teocriteo vedi Nisbet 2008, p.51. Dal significativo confronto con gli analoghi, per struttura ritmica e sintattica, b.7.44 ite domum pasti, si quis pudor, ite iuuenci ('Andate a casa sazi, se siete un po' discreti, andate giovenchi') e b.10.77 ite domum saturae, uenit Hesperus, ite capellae ('Andate a casa sazie, viene Espero, andate, caprette'), è evidente che «qui manca, dolorosamente, un moto a luogo come domum» (Cucchiarelli 2012, ad b.1.74): Melibeo invita le caprette a muoversi, non più, come sempre, verso casa, ma verso una meta lontana.

35. Anafora e geminatio sono spesso erroneamente assimilate, come nota Traina 1994, p.133. Sulla

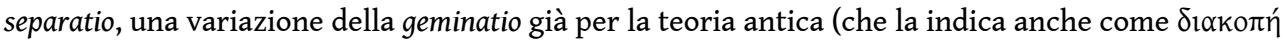

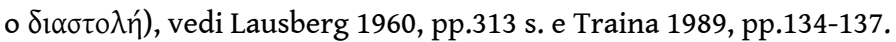

36. Se è vero che il diminutivo capella, l'unico frequente nelle Bucoliche (13 occorrenze, tutte in fine di verso), sembra essere preferito dai poeti rispetto alla forma base capra (prevalente in 
prosa) per motivi di eufonia, in questo passo non è assente una sfumatura affettiva. Si veda Marouzeau $1946^{2}$, p.168 e soprattutto la discussione di Zucchelli 1985, p.75.

37. Questo ordo verborum è noto anche come schema Cornelianum, in quanto si credeva fosse stato introdotto da Cornelio Gallo (è la tesi di Skutsch 1956, pp.198 s., ripresa nel recente contributo di Gagliardi 2017, pp.183-200, che considera questa particolare apposizione se non un'invenzione di certo un clichè della poesia di Gallo), si vedano l'articolo di Solodow 1986, pp.129-153, con esempi anche a partire da Archiloco, le osservazioni di Traina 1998, pp.11-17, che retrodata la figura a partire da Ennio (dub. V, I 6 Sk.), avanzando anche l'ipotesi di un caso in saturnio neviano (fr.1 Mor.), e ancora Landolfi 2011, pp.310-317 (su un probabile caso in Porcio Licinio).

38. Si veda Cucchiarelli 2012, ad loc. («Nell'agg. è il rimpianto per un passato florido, ora impossibile») e Bellincioni 1985, p.487, che a proposito del passo osserva che «con un'esclamazione nostalgica [...] al gregge è attribuito lo stato d'animo del pastore»), e in generale che l'aggettivo a volte «evoca una felicitas che sarebbe potuta essere ma non è stata». Felix (come del resto infelix) può veicolare un commento autoriale, come nei due casi di 'conditional makarismos' (per questa efficace definizione vedi Hardie 1994, ad Aen.9.337-338), dove è impiegato in un periodo ipotetico dell'irrealtà: Aen.9.335 ss. et iuuenem Serranum, illa qui plurima nocte / luserat, insignis facie, multoque iacebat / membra deo uictus: felix, si protinus illum / aequasset nocti ludum in lucemque tulisset ('e il giovane Serrano, che molto assai quella notte / aveva giocato, di nobile aspetto, e giaceva affranto le membra / dal soverchio iddio: fortunato, se continuo quel gioco / avesse agguagliato alla notte e protratto fino all'albore'), dove il poeta provoca il pathos mediante il riferimento alla giovane età (iuuenem) e alla bellezza dell'eroe (insignis facie), e Aen. 4.657 s. felix, heu nimium felix, si litora tantum / numquam Dardaniae tetigissent nostra carinae! ('felice, ahi troppo felice, se soltanto mai le dardanie / chiglie non avessero toccato le nostre spiagge'), dove l'effetto dell'aggettivo è rafforzato dalla separatio, dall'interiezione patetica heu e dall'avverbio nimium (sul sintagma heu nimium vedi Horsfall 2003, ad Aen.11.841).

39. Sulle apposizioni patetiche si veda Conte 2007, p.29 e Dainotti 2015, p.45, n.159 (con ulteriori esempi).

40. Al pathos dell'aggettivo felix si aggiunge quello dell'avverbio quondam, sul quale si veda Traina $2004^{2}$, ad loc. («l'avverbio del "tempo felice”, passato e perduto»), le note di Horsfall 2006, ad Aen. 3.14 e 3.49 (con materiale) e soprattutto Munzi 1988, pp.384 s.

41. Traina $2004^{2}$, ad loc: «nel quadro idillico dei vv.75-78, che richiama la serenità presente e futura di Titiro dei vv. 1-5 e 51-58, Melibeo è una presenza negata (anafora della negazione e del pronome personale, cf. v. 77: non me)».

42. Il poeta ricorre a un'apostrofe patetica anche per Pallante (Aen.10.507), Eurialo e Niso (Aen. 9.446 ss.), tutti giovani destinati ad una mors immatura. Vedi Traina $2004^{2}$, ad Aen.10.793.

43. Su questo nesso impiegato per introdurre una 'pathetic hope' (alla maniera dell'equivalente omerico eľ $\tau$ l५) vedi Harrison 1991, ad loc.

44. Sulla differenza semantica e stilistica tra sileo (indica il silenzio come assenza di suono e di movimento, ed è impiegato in contesti solenni e sacrali) e taceo (riferito al tacere umano, dice la volontarietà del silenzio) si veda Ricottilli 1990, p.7 e, sul passo in questione, p.11 (vedi anche Traina $2004^{2}$, ad loc.).

45. 1.18.11; $1.23 .9 ; 4.22 ; 4.23 .9$. Come nota Thomas 2011 , ad Hor.C.4.12.22, questi casi non sono riconducibili alla tendenza alla semplice giustapposizione pronominale ego te (non preceduta dalla negazione non), la quale, particolarmente comune in latino, è tuttavia impiegata da Orazio solo in S.2.6.60.

46. Sia in 1.18.11 ss. non ego te, candide Bassareu, / inuitum quatiam nec uariis obsita frondibus / sub diuum rapiam ('Tu non lo vuoi, Dioniso luminoso, / io non ti agiterò, ciò che si cela / tra il fogliame diverso / io non vorrò rapirlo nella luce') sia in 1.23 .9 s. atqui non ego te tigris ut aspera / Gaetulusue leo frangere persequor ('Ma non t'inseguo io come una tigre / feroce, un leone d'Africa, 
non voglio / infrangerti') è comunque possibile parlare di aversio. Per questi e gli altri passi tratti dalle Odi la traduzione è di E. Mandruzzato.

47. Questa forma di enjambement, che può essere definita 'interlinear juxtaposition' in quanto la giustapposizione di aggettivo e sostantivo è staccata tra due versi (sulla figura in Virgilio si veda Dainotti 2015, pp.31-46) non è infrequente in Orazio lirico, anche per via della misura più breve del verso (nella stessa ode ricorre ai vv.17, 19, 27, 30, 33, 40).

48. Per una dettagliata discussione sul tono ironico dell'encomio e sulla figura di Marco Lollio, console nel 21 a.C., si veda Thomas 2011, pp.196-198.

49. Sull'identificazione, a lunga discussa, del Virgilio dell'ode con il poeta mantovano si veda Thomas 2011, pp.226 s. e ad Hor.C.4.12.13 e ora anche Fedeli 2019, pp.1-12, che nega però tale interpretazione, osservando (p.12) che «la presenza di reminiscenze virgiliane in un carme diretto a un omonimo del poeta non costituirebbe una novità nella poesia augustea» ma piuttosto «una forma di scherzosa allusività».

50. Thomas 2011, ad loc. ben evidenzia l'effetto dell'elegante citazione virgiliana nella sequenza non ego te: «four times in the Odes $(9.30 ; 1.18 .11,23.9)$, and appropriately in addressing Virgil». Tra gli altri addentellati con la poesia di Virgilio è utile ricordare al verso 13 l'espressione adduxere sitim tempora ('la stagione ha portato la sete'), che, come nota Thomas 2011, ad loc., collocata subito prima dell'allocuzione a Virgilio, «inverts Virg. G. 3.482-3 sed ubi ignea uenis / omnibus acta sitis miseros adduxerat artos».

51. Vedi Peirano 2014, p.107. Non è impossibile (ma appare improbabile) una datazione al III sec. d.C.: se è vero, infatti, che un'attribuzione a Virgilio non è attestata prima di Donato, è anche vero che in un periodo di pieno alessandrinismo come il III secolo i poeti attingono non solo alla poesia augustea, ma anche a quella posteriore (ad esempio Stazio), della quale è difficile trovare chiare tracce nella Ciris. Si veda La Penna 2013, p.138.

52. La traduzione di questo e degli altri passi citati dalla Ciris è di P. Gatti.

53. Si potrebbe trattare di Messalla Corvino, soprattutto se si ipotizza che l'imitatore impersoni un Virgilio giovane che scrive a cavallo tra il 50 e il 40 a.C., un periodo in cui l'epillio era un genere particolarmente alla moda. Questa fiction cronologica spiegherebbe l'insolita scelta di questo genere ormai desueto nel primo o secondo secolo d.C., quando presumibilmente è stata scritta la Ciris. Vedi Peirano 2014, pp.107-109.

54. Sul pathos del lamento drammatico di Britomarti, una digressione patetica che costituisce un tratto caratterizzante dell'epillio latino rispetto a quello greco, si vedano le osservazioni di Perutelli 1979, pp.69-93.

55. Mai di pentametro. Maltby 2002, ad Tib.I, 6, 29 definisce appunto la sequenza «hexameter opening».

56. Le traduzioni dei passi tibulliani sono di M. Ramous.

57. Rhet. ad Her. 2.16.23 Concessio est, per quam nobis ignosci postulamus. Ea dividitur in purgationem et deprecationem. Purgatio est, cum consulto a nobis factum negamus. Ea dividitur in necessitudinem, fortunam, imprudentiam ('L'ammissione è quella per la quale chiediamo che ci si perdoni. Essa si divide in giustificazione e deprecazione. La giustificazione è quando affermiamo che non si è agito da noi intenzionalmente. Essa si divide in necessità, caso (fortuito), imprudenza.' Trad. F. Cancelli). Si veda Smith 1913, ad loc.

58. Am.1.8.28; Rem.495; Epist.10.67, 12.195; Fast.3.55, 6.763; Trist.1.8.37.

59. Le traduzioni dei passi tratti dalle Heroides sono di G. Rosati.

60. L'accorata negazione della colpa è simile a Aen.4.425 s. non ego cum Danais Troianam exscindere gentem / Aulide iuraui classemue ad Pergama misi ('Io non giurai con i Danai in Aulide di sterminare / la razza troiana o mandai navi mie verso Pergamo'), sul quale vedi Pease 1935, ad loc., che include il passo staziano tra i casi di aversio simili al verso virgiliano. Per il parallelo vedi anche Venini 1970, ad loc. 
61. L'episodio di Cilnio è paradigmatico della tecnica poetica di Silio, il quale è solito combinare $\mathrm{e}$ variare stilemi, temi e immagini virgiliane. Cilnio, un ostaggio in catene interrogato da un capo nemico, richiama la figura di Sinone (evidente l'addentellato anche nell'espressione nosces ... certamine $a b$ uno, citazione del virgiliano $a b$ uno disce omnis), sovvertendone l'ethos: non più un bugiardo ingannatore, ma un eroe desideroso di dire il vero per affrettare la sua morte. Per una fine analisi dell'episodio si veda Fernandelli 2012, pp. 144-153.

62. L'ammirazione di Ausonio nei confronti di Virgilio è del resto evidente dalla sua produzione, in particolare dal Cento nuptialis, un poemetto composto a partire da emistichi o versi virgiliani. Sulle diverse modalità con cui Ausonio 'riusa' Virgilio si veda Prete 1984, pp.422 s. (con bibliografia) e O’ Daly 2004, pp.141-154.

63. Accanto alla litote, si dovrà osservare che la citazione di Virgilio è ravvisabile anche nel rarissimo participio passato infletum, che richiama Aen.11.372 nos animae uiles, inhumata infletaque turba ('noi, anime da due soldi, plebe insepolta e illagrimata') - l'unica altra occorrenza prima di Ausonio è Val.Fl. 6.651 -, dove il termine è legato in allitterazione 'prefissale' ad inhumata, un altro participio che ben si attaglia al passo di Ausonio: il poeta afferma con forza che non rinuncerà a piangere suo figlio e, come suggerisce implicitamente la citazione, che non lo lascerà senza sepoltura, contravvenendo a quanto prescriveva la legge per i bambini morti nel primo anno di vita (su questo aspetto vedi Lolli 1997, ad Parentalia 10.4).

\section{RIASSUNTI}

By outlining a brief history of a poetic sequence, non ego te, since Plautus up until Ausonius, the author demonstrates that this word order, mainly a hexameter opening, far from being casual or simply ascribable to spoken language, is deliberately employed in passages symptomatically comparable for semantics and atmosphere (touching farewells or panegyrical apostrophes), in order to charge the diction with a strong pathetic nuance.

\section{INDICE}

Parole chiave : Poetic diction, pathos, apostrophe, Plautus, Catullus, Virgil, Horace

\section{AUTORE}

\section{PAOLO DAINOTTI}

Corpus Christi College, Oxford

Università di Napoli L'Orientale 